Classification

Physics Abstracts

$61.16 \mathrm{P}-68.20-73.40 \mathrm{M}-81.60 \mathrm{C}$

\title{
Monte-Carlo Simulations of Si Etching: Comparison with in-situ STM images
}

\author{
Philippe Allongue and Jérôme Kasparian* \\ Laboratoire de Physique des Liquides et Électrochimie, UPR 15 CNRS associé à l'Université P. \& M. \\ Curie, 4 Place Jussieu, F-75005 Paris, France
}

(Received July 4; accepted November 09, 1994)

\begin{abstract}
Résumé. - Limagerie par microscopie à effet tunnel (STM) in-situ est utilisée pour analyser quantitativement le décapage du $\mathrm{Si}$. On montre que l'on peut mesurer avec cette technique la vitesse d'attaque et l'anisotropie de la dissolution, deux paramètres importants en technologie, à partir de l'enregistrement de séquences d'images avec la résolution de marches atomiques. On montre aussi, pour la première fois, que les vitesses d'attaque sur les différents sites atomiques de surface (mono, di-hydrures de $\mathrm{Si}$ ) peuvent être déterminées en comparant des séquences d'images simulées de la dissolution du Si (méthode Monte-Carlo dans laquelle les atomes de Si sont arrachés au réseau de manière aléatoire) avec des séquences expérimentales.
\end{abstract}

\begin{abstract}
A quantitative analysis of Si etching is presented using in-situ real time STM imaging. It is shown that the rate and anisotropy of Si etching, two macroscopic parameters of interest in technology, can be determined from time sequences of images with the resolution of atomic steps on $\mathrm{n}$-Si(111). The relative reaction rates on the different atomic Si hydride sites available on the surface are also determined for the first time by comparing simulated (Monte-Carlo method in which Si atoms are randomly removed from the surface) with experimental sequences of STM images.
\end{abstract}

\section{Introduction.}

Within the last few years in-situ Scanning Probe Microscopies (SPM with $P=T$ and $F$ respectively for tunneling and force) have merged as major achievement in interfacial electrochemistry. The terminology in-situ refers here to the fact that the surface under observation is in contact and eventually reacts with an electrolyte. In the case of STM the approach uses the so-called 4electrode configuration which ensures that the tip current exclusively originates from a tunneling process between the tip and sample while the rate of the electrochemical reaction on the sample surface is controlled by a potentiostat. Real time in-situ STM imaging is now becoming an almost

* On leave for Laboratoire de Spectrométrie Ionique et Moléculaire, Université Claude-Bernard, Bât. 205, 43 Bd. du 11 novembre 1918, 69622 Villeurbanne Cedex, France. 
routine technique in electrochemical surface science and atomic resolution has been achieved on different substrates such as metal single crystals ( $\mathrm{Au}, \mathrm{Pt}, \mathrm{Cu}$ for instance) and semiconductors ( $\mathrm{Si}$, $\mathrm{Ge}$ and InSe). Two recent review articles cover the principles and recent achievements of in-situ STM or SFM on metals [1] and semiconductors [2].

Real time imaging offers the unique opportunity to visualize the dynamic of a chemical or a physical process and reach quantitative information about microscopic parameters, down to the atomic scale. In the UHV surface diffusion, 2-D phase transitions have been for instance investigated with the STM. By comparison, there is very little work in this direction at the solid/liquid interface although electrochemical interfaces offer the possibility of easily varying surface reactivity, coverage etc. by simply changing the potential applied to the sample.

The present paper is addressed to a quantitative analysis of Si etching, which is a usual step in microelectronic industry in several stages of the fabrication of integrated circuits. It is shown that semi-macroscopic parameters, such as the etch rate and reaction anisotropy, can be derived from sequences of in-situ STM images. In another part Monte-Carlo simulations of the reaction are presented. The comparison of simulated with experimental sequences of STM images is used for the first time to determine the reaction rate on the different surface atomic sites.

\section{In-situ STM results: $\mathrm{Si}(111)$ etching in $\mathrm{NaOH}$.}

The electrochemical response of $\mathrm{n}-\mathrm{Si}(111)$ in $\mathrm{NaOH}$ is shown in figure 1 . The rectifying behavior of the characteristics classically accounts for the diode-like behavior of the Si-liquid contact [3].

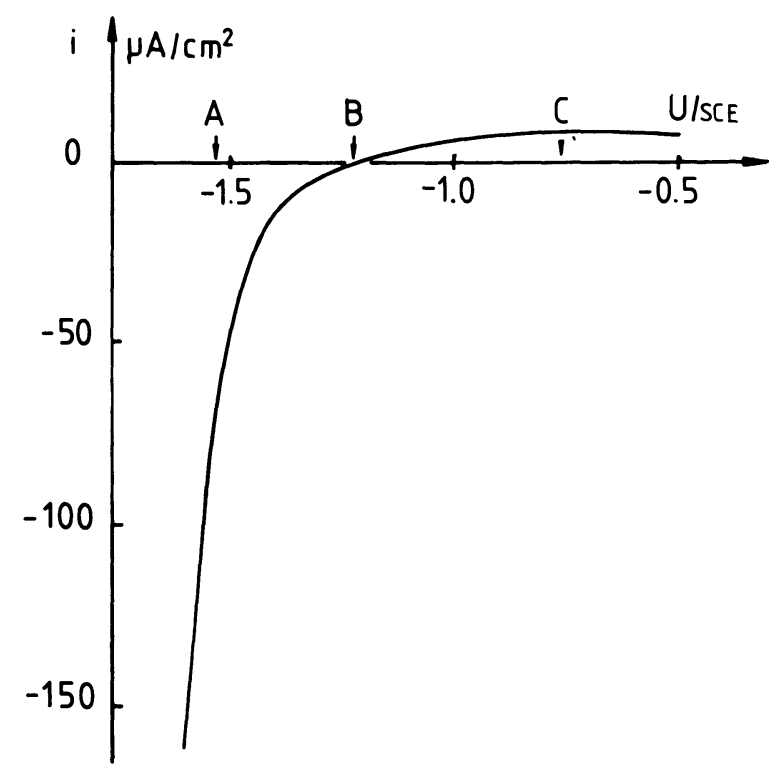

Fig. 1. - Electrochemical response of $\mathrm{n}-\mathrm{Si}(111)$ in $\mathrm{NaOH}$. The rectifying behavior stems from the diodelike behavior of the interface. A-C marks are the potentials were series of STM images have been taken.

Figure 2 presents three series of two STM images [4-5] showing how Si etching proceeds at potentials marked as $\mathrm{A}, \mathrm{B}$ and $\mathrm{C}$ in figure 1 . In series (a) the large density of electrons at the surface almost stops the dissolution. The series evidences however some etching which is completely 

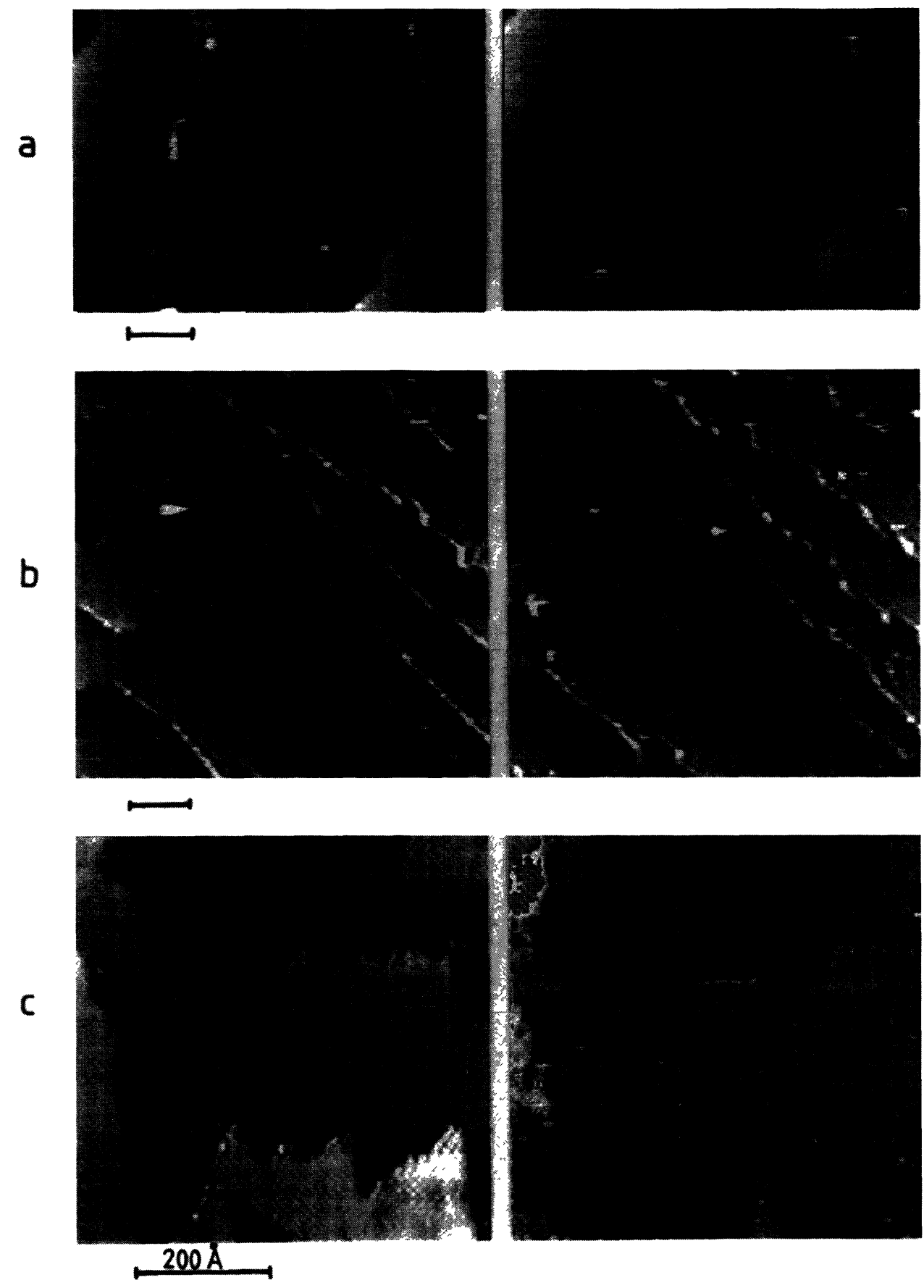

Fig. 2. - Three series of two in-situ STM images showing the dissolution of $\mathrm{n}-\mathrm{Si}(111)$ in $\mathrm{NaOH}$. Series (a): The two images are separated by $4 \mathrm{~min}$ and the sample was continuously at potential A (see Fig. 1). The dashed lines mark the initial position of steps. In series (b-c) the sample potential was set for $3 \mathrm{~s}$ at potential $\mathrm{B}$ and $\mathrm{C}$ between the two images which were recorded at potential $\mathrm{A}$. 
anisotropic since it exclusively occurs at step edges (all steps are one bi-layer high, i.e. $3.1 \AA \AA$ high) and no pit formation is observed on terraces during the 4 minutes of observation. The initial position of step edges is shown by the dashed line in the second image of series (a). Between the two images in series (b) the Si has been set for only $3 \mathrm{~s}$ at potential $\mathrm{B}$, which nearly corresponds to the usual situation in technology when the wafer is simply dipped in the etch bath. This procedure, also referred to as 'chemical etching' results in pronounced pitting of terraces (all pits are $3.1 \AA$ deep). In series (c) the sample has been also set at bias $C$, again for $3 \mathrm{~s}$, between the two images. Faster pit formation is observed due to anodic polarization. Note also that pits are nearly circular contrary to the triangular ones created in series (b).

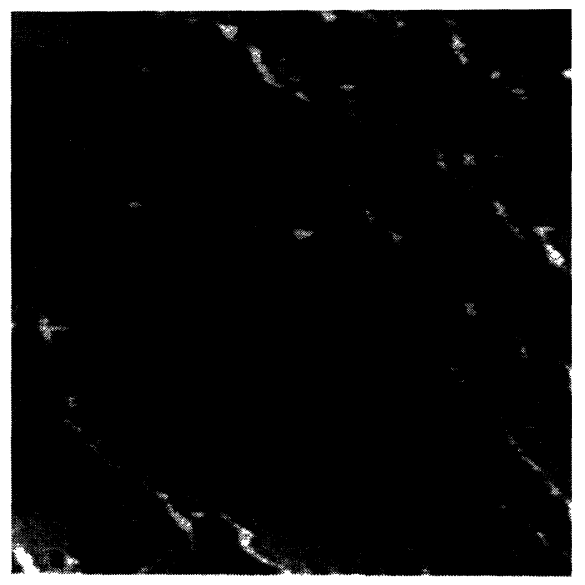

a

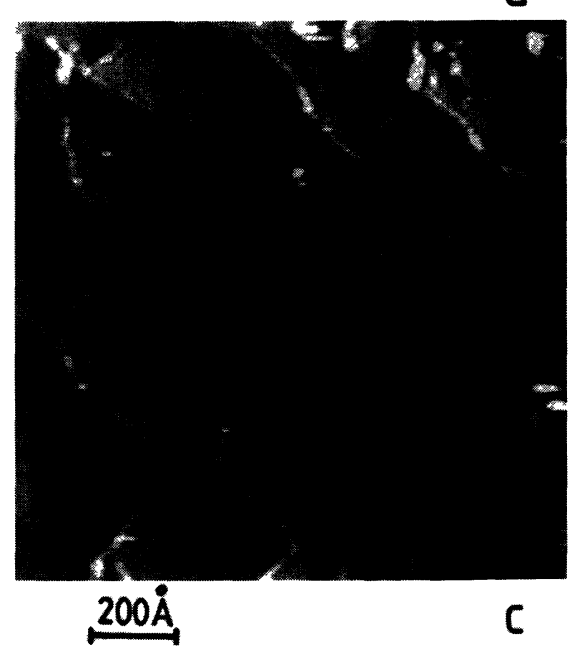

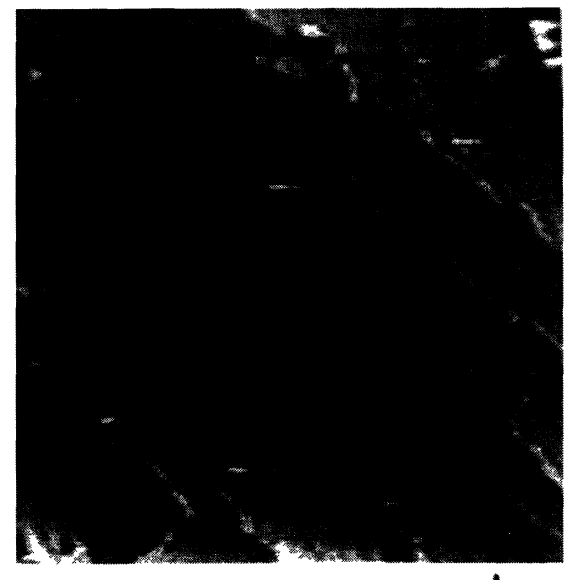

b

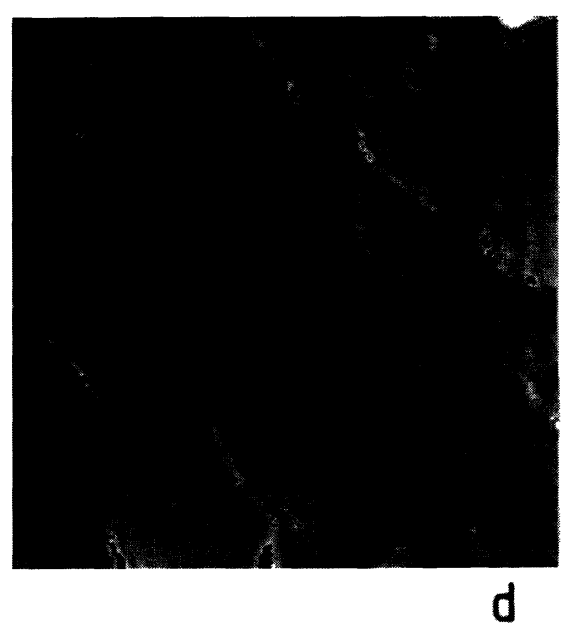

Fig. 3. - Time sequence of in-situ STM images showing the growth of pits at potential A. The series starts from the second image of series (b) of figure 2. Images (b-d) were recorded 38, 114 and $152 \mathrm{~s}$ after image (a).

Imaging the very beginning of pit formation is practically impossible experimentally with n-Si since long standing imaging is not possible in the corresponding electrochemical conditions $[2,4]$. 
In figure $2 b$ and $c$ the pits have been nucleated during the potential pulse while the regulation loop for the tip current was simultaneously opened and the tip immobilized [4]. If one operates at cathodic bias the event becomes so rare (see Fig. 1a) that imaging the initiation of nucleation is a matter of luck. Figure 3 studies the growth of etch pits immediately after their creation. This series will be used for simulation. It evidences that the triangular shape is preserved over a long period until pit coalesce or merge into step edges.

\section{Quantitative determinations from STM images.}

Several parameters accounting for micro- and macroscopic phenomenon can be measured from time sequences of STM images. The determination of the bias dependence of some of these parameters has yielded new insights into the dissolution mechanism of $\mathrm{Si}$ in $\mathrm{NaOH}$ [7]. The reader is referred to references $[4,5]$ for more details.

The first parameter of interest in technology is the vertical etch rates $R_{n}$. Its expression is given by $(\Delta S / S) \cdot h / \Delta t$, where $\Delta S$ is the surface area of terraces which has disappeared, including eventual pits, in the time interval $\Delta t, h$ is the step height and $S$ the total surface area under observation [the quantity $(\Delta S / S) \cdot h$ represents the volume of material removed per $\mathrm{cm}^{2}$ of sample since the process is a layer by layer dissolution]. Macroscopically $R_{n}$ is generally derived from losses of material and the result is averaged over several $\mathrm{mm}^{2}$ of sample. Although derived from ca $1000 \times 1000 \AA^{2}$ STM images the values of $R_{n}$ are surprisingly very consistent with macroscopic determinations [4], with the advantage that etch rates as small as $0.2 \AA / \mathrm{min}$ can be determined here while they are very difficult to measure accurately with macroscopic methods. The lateral etch rate $R_{l}$ is a second parameter of interest to characterize the reaction anisotropy. It is simply the speed at which step migrate. This is a typical microscopic parameter that can not be reached by other methods.

The anisotropy of the reaction can be defined as the ratio $R_{l} / R_{n}$. The larger this ratio the more anisotropic the etching. A macroscopic approach of the anisotropy is possible from etch rate measurements with samples of different orientations [6]. The frequency of etch pit generation is the last parameter which is directly attainable from STM images. This is a microscopic parameter since pits seen with other techniques are much deeper and larger due to the coalescence of smaller ones.

\section{Determination of the atomic reaction rates.}

The alterations that the 3-D ideal surface structure of a Si(111) surface (Fig. 4) undergoes with the applied potential (see Fig. 2) reflect changes in the (electro-)chemical reactivity of the different Si hydride species (mono-, di-, tri-hydrides), mostly in connection with variations of the surface density of electrons. It is indeed well known that the surface of Si remains completely $\mathrm{H}$ terminated, at all bias, as it has been explained by reaction models [7-9] and verified by ex-situ [10] as well as in-situ IR spectroscopy [11-12]. The purpose of this section is to show that the reaction rate on the different atomic sites at the surface are quantitatively attainable from the comparison of simulations with the above experiments.

Chemically, the dissolution proceeds by the hydrolysis of $\mathrm{Si}-\mathrm{H}$ bonds into a $\mathrm{Si}-\mathrm{OH}$ group as initial step. This polarizes the adjacent Si-Si back bonds and makes them unstable, which explains that $\mathrm{Si}-\mathrm{OH}$ groups are removed from the surface [7-9]. The rate of dissolution depends on several factors including the accessibility of $\mathrm{Si}-\mathrm{H}$ bonds to water molecules and the possibility to break the Si-Si back bonds. The strength of $\mathrm{Si}-\mathrm{H}$ bonds and its angle with the surface are important for 

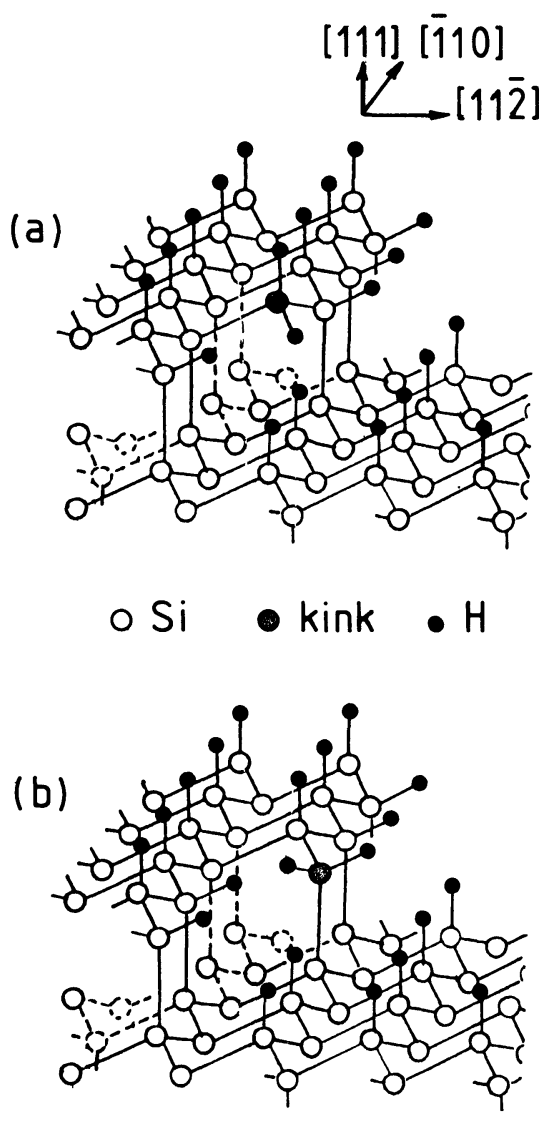

Fig. 4. - 3-D view of the $\mathrm{Si}(111)$ surface. Each $\mathrm{Si}$ surface atom (open circles) is bound to one or several $\mathrm{H}$ atoms (black circle) according to its coordination with the lattice. The kink site is grey shaded.

the hydrolysis step while steric hindrance governs the Si-Si bond breaking. Both factors explains that etching is favored at steps, especially when the reaction is chemical [7-9].

Physically, simulation (see Appendix) is based on the following assumptions. Silicon atoms are randomly removed from the surface at a rate $k_{i}$ (expressed in $\mathrm{s}^{-1}$ ) according to their lattice coordination. To simplify computation, we in fact consider three types of hexagonal sites centred on topmost atoms as shown in figure A1. Terrace sites (labelled as site T, reaction rate $k_{\mathrm{T}}$ ) which include only one vertical monohydride $\equiv \mathrm{Si}-\mathrm{H}$. Sites at step edges are of two kinds. The first ones (site $\mathrm{S}$, rate $k_{\mathrm{S}}$ ) correspond to an hexagonal cell with one vertical $\equiv \mathrm{Si}-\mathrm{H}$ and, on average, one "horizontal" step edge monohydride $\equiv \mathrm{Si}-\mathrm{H}$. The second type of step edge sites include either a vertical or an horizontal $\mathrm{Si}$ dihydride group $=\mathrm{Si}-\mathrm{H}_{2}$; They are considered as being equivalent in computations (site $\mathrm{U}$, rate $k_{\mathrm{U}}$ ). Given these definitions and the mechanism of etching the dissolution rates $k^{\prime}$ s are expected to be in the series $k_{\mathrm{T}}<k_{\mathrm{S}}<k_{\mathrm{U}}$. Computed images correspond to $576 \times 576 \AA^{2}$ which is about one fourth of STM images (Fig. 2).

Figure 5 is a simulated time sequence showing the attack of a (111) terrace initially virgin of any defect. The reaction rates are $k_{\mathrm{T}}=10^{-4} \mathrm{~s}^{-1}, k_{\mathrm{S}}=10^{-2} \mathrm{~s}^{-1}$ and $k_{\mathrm{U}}=1 \mathrm{~s}^{-1}$. The attack proceeds with the nucleation and growth of triangular pits which evidences the three possible orientations, parallel to the $[1 \overline{1} 0]$ directions, of stable $\equiv \mathrm{Si}-\mathrm{H}$ terminated step. The visual analogy between 


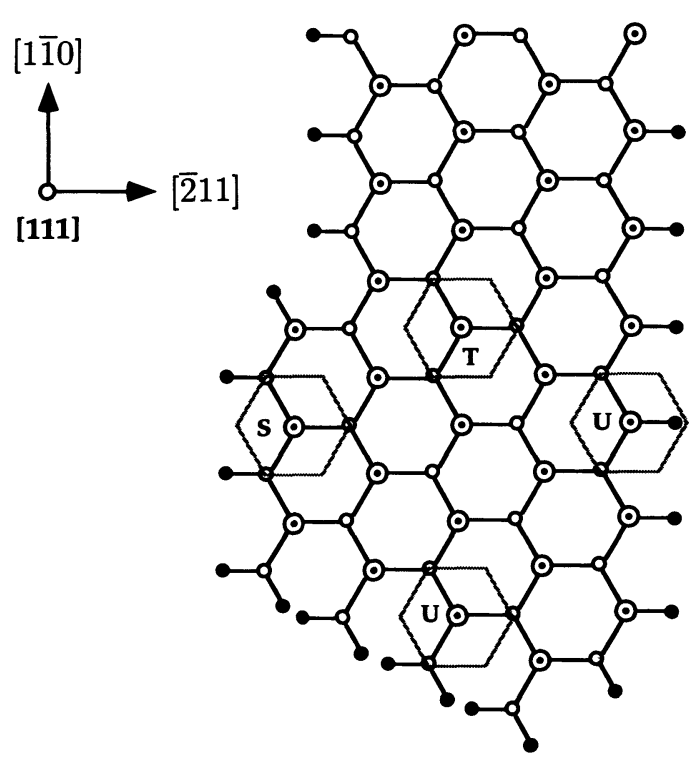

Fig. A1. - Top view of the $\mathrm{Si}(111)$ surface defining the different atomic sites concerned in simulations. Each of them is an hexagon marked by a letter T, $\mathrm{S}$ and $\mathrm{U}$, respectively for vertical terrace $\equiv \mathrm{SiH}, \mathrm{step} \equiv \mathrm{SiH}$ and step $=\mathrm{SiH}_{2}$ silicon hydride species.

simulations and STM images of figure 3 qualitatively validates the model. Figure 6 concerns the dissolution at step edges. No pitting was allowed on terraces $\left(k_{\mathrm{T}}=0\right)$ to compare results with the sequence in figure $2 \mathrm{a}$. The simulation was started after a row of sites, parallel to the [110], has been artificially removed so as to initiate the attack at $\mathrm{a} \equiv \mathrm{Si}-\mathrm{H}$ terminated step and at anstable $=\mathrm{Si}$ $\mathrm{H}_{2}$ terminated one, respectively exclusively composed of $\mathrm{S}$ sites on the right and of $\mathrm{U}$ sites on the left side of arrows. In accordance with expectations the $=\mathrm{SiH}_{2}$ terminated step becomes rapidly rougher and migrates faster than the $\equiv \mathrm{Si}-\mathrm{H}$ terminated one which remains almost straight. Note that a straight portion, corresponding to $\mathrm{a} \equiv \mathrm{Si}-\mathrm{H}$ terminated step, is slowly appearing on the left step. Parameters were $k_{\mathrm{S}}=10^{-2} \mathrm{~s}^{-1}$ and $k_{\mathrm{U}}=1 \mathrm{~s}^{-1}$ as in figure 5 .

\section{Discussion.}

5.1 COMPARISON WITH EXPERIMENT. - Figure 7 shows the time dependence of the etch rate for different simulations. In the case of the attack at steps (Fig. 6) the etch rate was calculated as exposed in section 2 with the surface under observation being the surface of the entire cell. The dissolution proceeds at a constant rate. That the ratio of speeds $R_{l}$ with which the two types of steps are migrating is only $\approx 5$ although the ratio $k_{\mathrm{U}} / k_{\mathrm{S}}$ was set to 100 demonstrates that kink sites are governing the dissolution on "stable steps". With increasing probability $k_{\mathrm{S}}$ the stable step move faster and becomes rougher.

In the case of the growth of pits, such as in the STM experiment of figure 3, the etch rate was calculated by subtracting the image obtained at time $t$ with the initial one. In the case of figure 3 the first image was taken as reference. To compare experimental data with simulations the origin of computed times was taken such that the size of simulated pits would make them easily visible in STM images [e.g. $t=59 \mathrm{~s}$ was taken as origin of times in the case of Fig. 5].

Figure 7 evidences an exponential time dependence of the initial growth of pit, both in sim- 


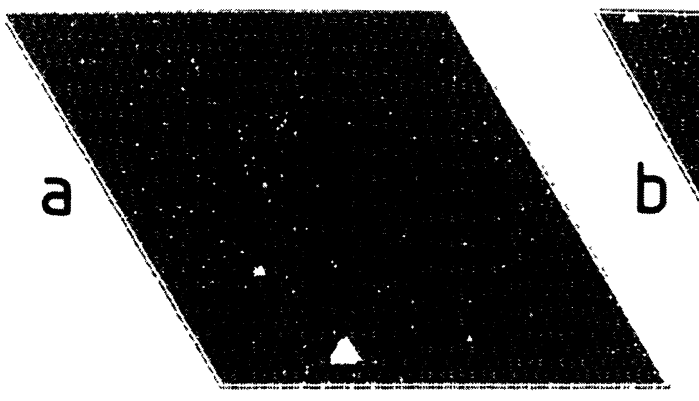

59

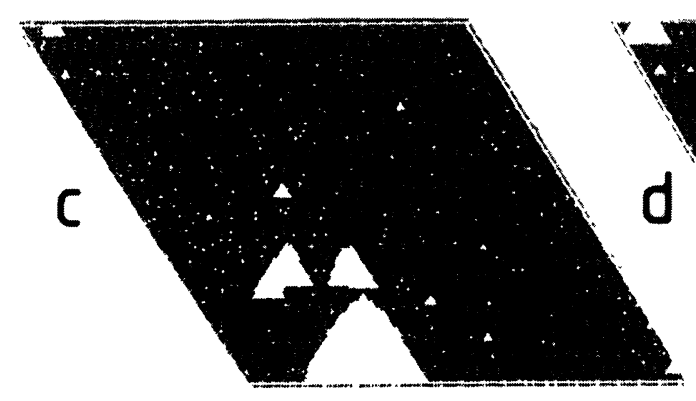

130

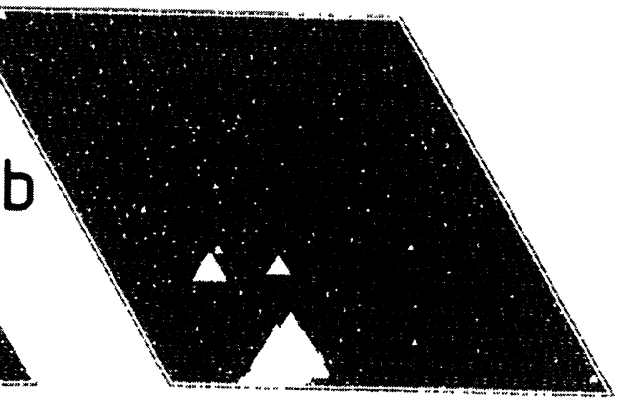

100

Fig. 5. - Simulated time sequence of images showing the nucleation and growth of pits on a virgin terrace. Parameters are $k_{\mathrm{T}}=10^{-4} \mathrm{~s}^{-1}, k_{\mathrm{S}}=10^{-2} \mathrm{~s}^{-1}$ and $k_{\mathrm{U}}=1 \mathrm{~s}^{-1}$.

ulations (curves a and b) and experimentally $(\bullet)$ which further justifies our model. A relative agreement with STM data is obtained with $5 \times 10^{-5}<k_{\mathrm{T}}<10^{-4} \mathrm{~s}^{-1}, k_{\mathrm{S}}=10^{-2} \mathrm{~s}^{-1}$ and $k_{\mathrm{U}}=1$ $\mathrm{s}^{-1}$. The uncertainty in the determination of $k_{\mathrm{T}}$ stems from the fact that the etch rate derived from STM images includes the attack at steps in contrast to simulations where pits are solely considered. The number of Si layers exposed to solution being about $4-5$ on average in $1400 \times 1400 \AA^{2}$ STM images (Fig. 2) and simulated images being one fourth of STM images, experimental data can be better accounted for by adding the etch rate at one step to the growth of pits, as in figure 5 . This is demonstrated by curve $(+)$ in figure 7 which is the sum of curve (b) and the average $(0.3$ $\AA / \mathrm{min}$ ) of etch rates at " $=\mathrm{SiH}_{2}$ " and " $\equiv \mathrm{SiH}$ " steps (see above curves c and d of Fig. 7).

The applied potential has a drastic effect of the dissolution rates. For instance, simulating the time sequence shown in figure $2 \mathrm{c}$, requires $k_{\mathrm{T}}=0.01 \mathrm{~s}^{-1}, k_{\mathrm{U}}=1 \mathrm{~s}^{-1}$ and $k_{\mathrm{S}}=0.5 \mathrm{~s}^{-1}$, which illustrates the activation by potential of the electrochemical reaction on the most stable atoms (the rate $k_{\mathrm{T}}$ is multiplied by a factor $5 \times 10^{3}$ and $k_{\mathrm{U}}$ by 50 ).

5.2 INTERPRETATION OF SIMULATION. - Given the quantitative agreement between simulations and STM images it can be concluded that Si etching actually occurs according to the random removal of atoms from the surface. No surface diffusion needs being considered, which is consistent with the covalent nature of $\mathrm{Si}$.

It is very interesting to notice in simulated images that isolated missing terrace sites do not necessarily lead to immediate growth of a pit. Such "point defects" look stable upon long periods of time. The rate of pit growth really increases if $2-3$ other terrace sites are attacked next to 

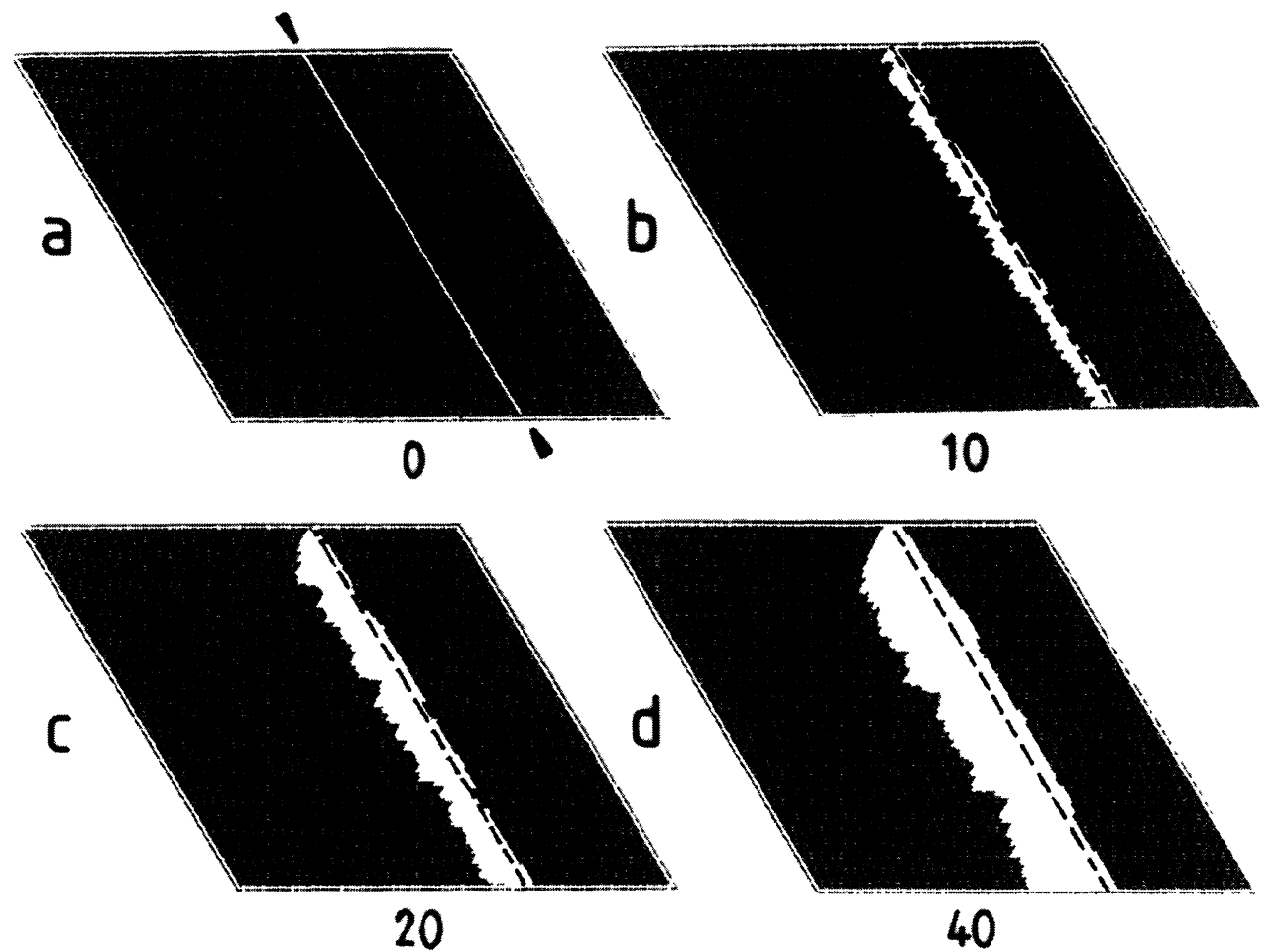

Fig. 6. - Simulated time sequence of images showing the attack at two steps. The one on the left is $=\mathrm{SiH}_{2}$ terminated (unstable step) and that on the right is $\equiv \mathrm{SiH}$ terminated (stable step). Arrows give the position of the row of atoms removed before experiment. Parameters are $k_{\mathrm{S}}=10^{-2} \mathrm{~s}^{-1}$ and $k_{\mathrm{U}}=1 \mathrm{~s}^{-1}$ and $k_{\mathrm{T}}=0$ $\mathrm{s}^{-1}$ (no pitting allowed).

the first one. This result fits very well reality if one considers that the "attack" of the very first terrace site corresponds actually to the hydrolysis step of the vertical $\equiv \mathrm{Si}-\mathrm{H}$ monohydride, the subsequent attack on neighbouring sites corresponding also to $\mathrm{Si}-\mathrm{OH}$ formation followed by rapid removal of the $2-3$ terrace $\equiv \mathrm{Si}-\mathrm{OH}$. That several neighbouring $\equiv \mathrm{Si}-\mathrm{OH}$ groups are necessary to initiate a pit agrees with the picture that the process requires to overcome a great energy barrier to break the Si-Si back bonds underneath $\mathrm{a} \equiv \mathrm{Si}-\mathrm{OH}$ group. Large strains are expected when comparing the hard sphere diameter of a water molecule $(2.9 \AA)$ and the Si-Si bond length $(2.35$ $\AA$ ). The surface needs being made unstable by strong polarization of the Si-Si back bonds [7]. This observation is supported by the fact that $\mathrm{Si}-\mathrm{OH}$ bonds (which are precursor to dissolution) have been recently identified by in-situ STM after the surface was held at the rest potential for a short period of time [13].

\section{Conclusion.}

In summary, we have presented a quantitative analysis of time sequences of in-situ STM images showing $\mathrm{Si}$ etching with the resolution of atomic steps. The comparison of experiments with Monte-Carlo simulation demonstrates that the dissolution of Si proceeds according to the random removal of Si atoms from the surface. Reaction rates on the atomic scale have been determined 


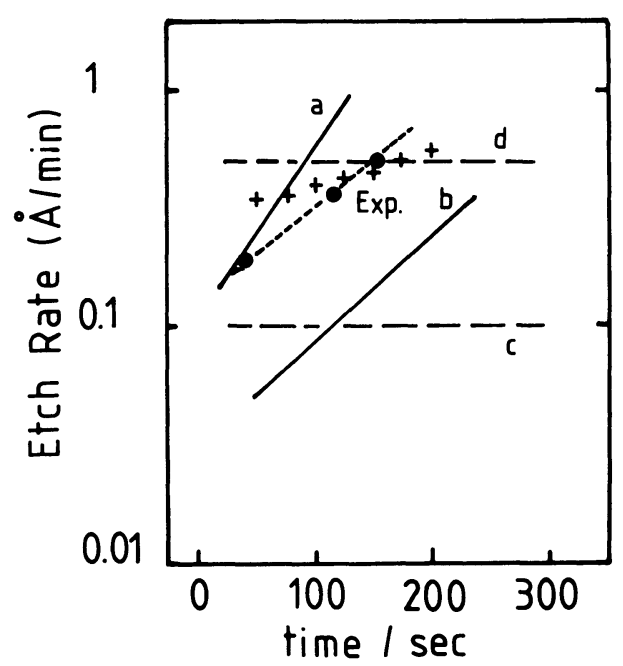

Fig. 7. - Simulated and experimental time dependence of the etch rate. Experimental $(\bullet)$ data are derived from figure 3. Curve (a) corresponds to figure $5\left(k_{\mathrm{T}}=10^{-4} \mathrm{~s}^{-1}\right)$ and curve (b) is calculated with $k_{\mathrm{T}}=510^{-5}$ $\mathrm{s}^{-1}\left(k_{\mathrm{S}}=10^{-2} \mathrm{~s}^{-1}\right.$ and $k_{\mathrm{U}}=1 \mathrm{~s}^{-1}$ in both cases). Curves (c) and (d) correspond to the attack at $\equiv \mathrm{SiH}$ and $=\mathrm{SiH}_{2}$ terminated steps $\left(k_{\mathrm{T}}=0 \mathrm{~s}^{-1}, k_{\mathrm{S}}=10^{-2} \mathrm{~s}^{-1}\right.$ and $\left.k_{\mathrm{U}}=1 \mathrm{~s}^{-1}\right)$. Curve $(+)$ is the sum of curve (b) with the average of etch rates at steps.

for the first time with this method. Results evidence that the reaction rate on kink sites is mostly governing the macroscopic speed of the reaction and that the bias has a drastic effect on other sites.

\section{Appendix}

Physically, at time $t$, the surface consists in $n_{i}(t)$ atoms of type $i$ whose rate of dissolution (constant $k_{i}$, expressed in $\mathrm{s}^{-1}$ ) is only depending on their lattice coordination. The total dissolution rate of the surface being therefore simply given by the sum $\sum(i) k_{i} n_{i}(t)$, one atom is removed, on average, during the time interval $d t=1 /\left(\sum(i) k_{i} n_{i}(t)\right)$.

Simulation consist in the successive random removal of hexagonal sites $\mathrm{Si}$ atomic sites shown in figure A1. To simplify three types of sites are considered. The time increment in simulation is taken as $\Delta t=1 /\left(\sum(i=T, S, U) k_{i} n_{i}(t)\right)\left(\Delta t\right.$ is not constant since the populations $n_{i}$ vary with time) and we consider that exactly one atom is removed from the surface during $\Delta t$ (this avoids unsuccessful attempts). Statistics concerns however which atom is removed. This is done at random in two stages.

The type of the site is first defined by establishing the three populations $n_{i}(t)$ and ratio $v_{i}=$ $k_{i} n_{i}(t) /\left(\sum(j=T, S, U) k_{j} n_{j}(t)\right)$ which represent the probability $\left(0 \leq v_{i} \leq 1\right)$ to remove a site of type $i$ between $t$ and $t+\Delta t$. Physically $v_{i}$ represents the (normalized) partial speeds of the reaction on all sites of type $i$. Choosing at random a number $x(0 \leq x \leq 1)$, designs the family $j$ if $\sum(i<j)\left(v_{i}\right) \leq x<\sum(i \leq j)\left(v_{i}\right)$. After this stage, the atomic site which is actually attacked is then chosen, also at random among a list of the $n_{j}(t)$ sites of type $j$ (they are all equiprobable). Given the foregoing definitions, it is important to notice that the $k^{\prime} s$ may be greater than unity. 


\section{References}

[1] Siegenthaler H., in "Scanning Tunneling Microscopy II", R. Wiesendanger and H.-J. Güntherodt Eds., Springer Series Surf. Sci. 28 (1992).

[2] Allongue P., in "Advances in Electrochemical Science and Engineering", H. Gerischer and C. W. Tobias Eds., Vol. 4, Weinheim, in press.

[3] Morrison S.R., in “Electrochemistry at Semiconductor and Oxidized Metal Electrodes" (Plenum Press, New York, 1980).

[4] Allongue P., Kieling V. and Gerischer H., J. Electrochem. Soc. 140 (1993) 1009.

[5] Allongue P., Brune H. and Gerischer H., Surf. Sci. 275 (1992) 414.

[6] Kendall D.L., Ann. Rev. Mater Sci. 9 (1979) 373 and Seidel H., Csepregi L., Heuberger A. and Baumgärtel H., J. Electrochem. Soc. 137 (1990) 3612 and 3626.

[7] Allongue P., Kieling V. and Gerischer H., J. Electrochem. Soc. 140 (1993) 1018.

[8] Gerischer H., Allongue P. and Costa-Kieling V., Ber. Bunsenges. Phys. Chem. 97 (1993) 753.

[9] Allongue P., Kieling V. and Gerischer H., Electrochim. Acta, in press.

[10] For a review, see Higashi G.S. and Chabal Y.J., in "Handbook of Semiconductor Wafer Cleaning Technology", W. Kern Ed. (Noyes Publications, Park Ridge, 1993).

[11] Venkateswara Rao A., Ozanam F. and Chazalviel J.-N., J. Electrochem. Soc. 138 (1991) 153.

[12] Rappich J., Lewerenz H.J. and Gerischer H., J. Electrochem. Soc. 141 (1994) L187.

[13] Allongue P., to be published. 